\title{
PREVALENCE OF OPEN ANGLE GLAUCOMA IN ACCOMPANYING FIRST DEGREE RELATIVES OF PATIENTS WITH GLAUCOMA
}

\author{
Franciele Vegini, Natanael Figueiroa Filho, Raphael Furlan Lenci, Diogo \\ Garcia Neto, Remo Susanna Junior
}

doi: $10.1590 / \mathrm{S1807-59322008000300007}$

Vegini F, Figueiroa-Filho N, Lenci RF, Garcia-Neto D, Susanna-Junior R. Prevalence of open angle glaucoma in accompanying first degree relatives of patients with glaucoma. Clinics. 2008;63:329-32.

OBJECTIVE: The aim of this study was to determine the prevalence of open angle glaucoma in first-degree relatives accompanying POAG patients during routine examination in a reference hospital.

METHOD: First-degree relatives of primary open angle glaucoma patients who accompanied their relatives to the glaucoma service of a reference hospital were screened for glaucoma.

RESULTS: One-hundred and one first-degree relatives were examined, of which $56.4 \%$ had never had their intraocular pressure measured. $10.9 \%$ had previously been diagnosed with glaucoma, and $5.9 \%$ were newly diagnosed during this study.

CONCLUSIONS: The eye examination of first-degree relatives identified a significant percentage of individuals with glaucoma. Despite being first-degree relatives of glaucoma patients, 56.4\% of the companions had never had their eye pressure measured, demonstrating a lack of awareness about this disease.

KEYWORDS: Glaucoma. Relatives. Family. Risk. Prevalence.

\section{INTRODUCTION}

Primary open angle glaucoma (POAG) is a progressive optic neuropathy with characteristic structural changes in the optic disc, frequently associated with corresponding changes in the visual field. ${ }^{1}$ It has been identified as one of the most important causes of irreversible vision loss, mainly in African-Americans, in whom it is responsible for up to $19 \%$ of blindness in adults over 40 years of age. ${ }^{2,3}$

Although the initial events of the disease are not yet completely understood, ${ }^{4,5}$ it is possible that genetic changes could be responsible for the initiation of a sequence of events that lead to a higher resistance in aqueous humor drainage, as well as a higher optic disc head susceptibility to certain levels of intraocular pressure (IOP). ${ }^{4}$

Hospital das Clínicas da Faculdade de Medicina da Universidade de São Paulo - São Paulo/SP, Brazil.

franciveg@hotmail.com

Received for publication on December 16, 2007

Accepted for publication on February 19, 2008
New advances in the genetic understanding of glaucoma have been postulated recently. ${ }^{6,7}$ Positive family history is an important risk factor, and previous studies indicate that approximately 5\% of POAG results from mutations in the myocilin (MYOC) gene, raising the possibility of identifying individuals who are genetically predisposed to glaucoma..$^{9,10,11}$ The relative risk of developing POAG in a population with a positive family history is $9.2 .{ }^{12}$ The presence of a family history of glaucoma is a risk factor for progression from ocular hypertension to POAG. ${ }^{9}, 12$ However, routine untargeted genetic testing for MYOC mutations in patients with POAG are of limited value until additional significant genetic risk factors are identified. ${ }^{1,10}$

Epidemiologic findings show that other risk factors include a family history of POAG, higher intraocular pressure (IOP), age, ethnic origin, and diagnosis with diabetes mellitus. ${ }^{5}$ Approximately half of POAG patients have a family history of glaucoma. ${ }^{1}$ Early detection of the disease is critical in halting glaucomatous damage and in minimizing irreversible vision loss. ${ }^{13}$ Adjusted analysis 
revealed that a family history of glaucoma in first-degree relatives and screenings measuring an IOP $>21 \mathrm{~mm} \mathrm{Hg}$ have statistically significant associations with the development of glaucoma. ${ }^{13} \mathrm{~A}$ combination of these findings gave an overall sensitivity of $81.7 \%$ with a specificity of $55 \% .^{13}$

The aim of this study was to determine the prevalence of open angle glaucoma in first-degree relatives accompanying POAG patients during routine examination in a reference hospital.

\section{MATERIALS AND METHODS}

This was a prospective, population-based study, approved by the hospital Institutional Review Board and Joint Committee for Clinical Investigation. All first-degree family members who were accompanying their relatives to the glaucoma service had a full ophthalmologic examination. Every participant was asked if they were already in treatment for glaucoma and if they had previously had their intraocular pressure taken. The inclusion criteria for the study were: first-degree relatives of a glaucoma patient, age greater than 30 years, and accompaniment of a POAG patient to the glaucoma service at a reference hospital. Families with closed or narrowed angles and secondary glaucoma were excluded from the study.

The optic disk was evaluated by indirect ophthalmoscopy with a 78 dioptric lens and the intraocular pressure (IOP) level was measured by Goldmann applanation tonometry. The patients who had an IOP under $21 \mathrm{mmHg}$ without medication and the absence of typical optic disk neuropathy were considered normal $(\mathrm{N})$ and were advised to have an annual check-up as follow up. Those who had an IOP level greater than or equal to $21 \mathrm{mmHg}$ in either eye in the absence of typical optic disk neuropathy were classified as glaucoma suspects (S) and referred to visual field testing (Sita Standard automated static perimetry 24-2 program on the Humphrey Visual Field Analyzer [Zeiss-Humphrey Systems, Dublin, CA]). This test was used to determine if a patient had glaucomatous visual field defect. According to Andersons' criteria, ${ }^{14}$ glaucomatous visual field defect is characterized by glaucoma hemifield test results outside the normal limits, a corrected pattern standard deviation (CPSD) with a probability less than $5 \%$, or a cluster of 3 or more points in the pattern deviation plot with a probability less than $5 \%$, one of which must have a probability level less than $1 \%$. As CPSD is not available in Sita, we used the PSD criteria to establish a diagnosis of glaucomatous visual field defects. Patients were submitted to another visual field test one month after the first exam to confirm the defect. Only patients with reproducible visual field defects were considered glaucomatous patients. Non-glaucomatous visual field defects were excluded.

Individuals were diagnosed with POAG if they fulfilled at least two of the following criteria: (1) typical glaucomatous visual field defect and greater or equal to a 0.7 optic cup/ disc ratio in the affected eye or equal or grater than 0.3 asymmetry between both eyes; (2) glaucomatous typical optic disk damage, such as optic disk hemorrhages, notching or a localized or diffuse nerve fiber layer defect; or (3) an intraocular pressure greater than or equal to $22 \mathrm{~mm} \mathrm{Hg}$ in either eye. Once the diagnosis was made, all of the patients had a complete eye examination, including gonioscopy. The patients who were diagnosed $(\mathrm{G})$ were referred to the reference hospital for glaucoma treatment.

\section{RESULTS}

One hundred and one first-degree relatives of patients with glaucoma were examined. Of these, $84(83.1 \%)$ were female and $17(16.9 \%)$ were male. The average age was 48.2 years old $( \pm 11.15)$, and $57(56.4 \%)$ were white, $22(21.8 \%)$ were Hispanic, 20 (19.8\%) were black and $2(2 \%)$ were of Asian origin.

Forty-four of the relatives (43.6\%) had already been examined for glaucoma. Of these, 36 (42.8\% of the women) were female and eight ( $47.0 \%$ of the men) were male. Fiftyseven (56.4\%; confidence interval 46.2\%-66.3\%) had never had their intraocular pressure measured; of these, 48 (57.2\% of the women) were female and nine (53\% of the men) were male. Among those examined, 11 (10.9\%; confidence interval $5.6 \%-18.7 \%$ ) had previously been diagnosed with glaucoma and were undergoing treatment. Of these eleven patients, nine were offspring and two were siblings.

A new diagnosis of glaucoma was made in six $(5.9 \%$; confidence interval 2.2\%-12.5\%) of the examined patients. Of these six new cases, four were offspring and two were siblings. Four (3.9\%; confidence interval 1.1\%-9.8\%) were suspected to have glaucoma and $80(79.3 \%$; confidence interval 70\%-86.6\%) had no sign of glaucoma.

\section{DISCUSSION}

Although many civic organizations have taken the initiative to provide mass glaucoma screening programs, an efficient, cost-effective screening protocol has yet to be defined..$^{13}$ One possible strategy for enhancing the effectiveness of glaucoma screening protocols is to focus on a high-risk population, ${ }^{13}$ such as those who have a family history of the disease.

Many studies have investigated relatives of POAG patients to identify signs of the disease. ${ }^{15,16}$ It is estimated that less than half of affected people in developed countries 
are aware of their diagnosis. ${ }^{13}$ The Glaucoma Inheritance Study in Tasmania and other states in Australia examined 442 individuals from five pedigrees with a strong positive family history of POAG (not only first degree relatives), $13 \%$ had a prior diagnosis of POAG or glaucoma-suspect status, and $16 \%$ were newly diagnosed. ${ }^{9}$ In our study, $10.9 \%$ had a prior diagnosis, and $9.8 \%$ were newly diagnosed as POAG or glaucoma suspects. It is likely that this number would be even greater if all of the relatives were examined, but that was not the purpose of our study. Another finding of the study in Tasmania was that $27 \%$ of POAG patients with a family history of POAG were completely unaware of their family history, mostly in those who had distant relatives affected. ${ }^{9}$

In another population-based familial aggregation study, the prevalence of glaucoma was $10.4 \%$ in the siblings of patients, $1.1 \%$ in the offspring of patients, $0.7 \%$ in the siblings of controls, and $0 \%$ in the offspring of controls. ${ }^{5}$ The lifetime risk of glaucoma was $22 \%$ in relatives of patients with glaucoma, almost ten times higher than in controls, suggesting that at least one sixth of all glaucoma cases in the general population may be caused by a genetic component. ${ }^{5}$
Epidemiologic findings in the Baltimore Eye Survey maintained that POAG family history indicates an important risk factor for disease development. ${ }^{17}$ The Barbados Eye Study also suggests that people more vulnerable to developing POAG are elderly males with a family history of glaucoma. ${ }^{18}$ However, these two studies were almost exclusively performed with observational data and oral questionnaires for hereditary confirmation. Randall L et al. published a study in which 86 relatives of POAG patients were examined..$^{19}$ The prevalence of the disease in relatives was $30 \%$, as diagnosed by intraocular pressure values and automated perimetry defects. ${ }^{19}$ When POAG is present in more than one family member, immediate as well as more distant relatives should be evaluated for glaucoma by means of clinical examination and automated perimetry. ${ }^{19}$

The present study identified a significant percentage of first-degree relatives of glaucoma patients with glaucoma. Despite being first-degree relatives of glaucoma patients, $56.4 \%$ of companions had never had their eye pressure measured, depicting a lack of awareness about the disease. The results of this study reveal the need to take action to increase the awareness of the disease among relatives of glaucoma patients.

\section{REFERENCES}

1. Susanna JR, Weinreb RN. Os pacientes com glaucoma devem se submeter a um teste genético? In: Glaucoma: perguntas \& respostas. Rio de Janeiro: Cultura Médica; 2005.

2. Leske MC. The epidemiology of open-angle glaucoma: a review. Am J Epidemiol. 1983;118:166-90.

3. Sommer A, Tielsch JM, Katz J. Racial differences in the causespecific prevalence of blindness in East Baltimore. N Engl J Med. 1991;395:1412-17.

4. Allingham RR, Damji D, Freedman S, Moroi S, Shafranov G. Shields' Textbook of Glaucoma. $5^{\text {th }}$ edition. Philadelphia: Lippincott Williams \& Wilkins; 2005.

5. Wolfs RCW, Klaver CCW, Ramrattan RS, Van Duijn CM, Hofman A, de Jong PTVM. Genetic risk of primary open-angle glaucoma: populationbased familial aggregation study. Archives of Ophthalmology. 1998;116:1640-5.
6. Lichter PR. Genetic clues to glaucoma's secrets. The L Edward Jackson Memorial Lecture. Part 2. Am J Ophthalmol. 1994;117:706-27.

7. Sarfarazi M. Recent advances in molecular genetics of glaucomas. Hum Mol Genet. 1997;6:1667-77.

8. Quigley HA. The search for glaucoma genes-implications for pathogenesis and disease detection [editorial]. N Engl J Med.1998;338:1063-64.

9. McNaught AI, Allen JG, Healey DL, Coote MA. Accuracy and Implications of a reported family history of glaucoma: experience from the glaucoma inheritance study in Tasmania. Archives of Ophthalmology. 2000;118:900-4.

10. Frezzotti R, Renieri A, Frezzotti P. Adult-onset primary glaucoma and molecular genetics: a review. European Journal of Ophthalmology. 2004;14:220-5. 
11. Wiggs JL, Allingham RR, Vollrath D. Prevalence of mutations in TIGR/Myocilin in patients with adult and juvenile primary open-angle glaucoma. Am J Hum Genet. 1998;63:49-52.

12. Wu J, Hewitt AW, Green CM, Ring MA, McCartney PJ, Craig JE, et al Disease severity of familial glaucoma compared with sporadic glaucoma. Archives of Ophthalmology. 2006;124:950-4.

13. Vistamehr S, Shelsta HN, Palmisano PC, Filardo G, Bashford K, Chaudhri K, et al. Glaucoma screening in a high-risk population. Journal of Glaucoma. 2006;15:534-40.

14. Anderson DR, Patella VM. In: Automated Static Perimetry. St Louis: Mosby; 1999.

15. Miller SJH. Genetics of glaucoma and family studies. Trans Ophthalmol Soc UK. 1978;98:290-92.
16. Rosenthal AR, Perkins ES. Family studies in glaucoma. Br J Ophthalmol. 1985;69:664- 67.

17. Tielsch JM, Katz J, Sommer A. Family history and the risk of primary open angle glaucoma. The Baltimore Eye Survey. Arch Ophthalmol. 1994;112:69-73.

18. Nemesure B, Leske MC, He Q, Mendell N. Analyses of reported family history of glaucoma: a preliminary investigation. The Barbados Eye Study Group. Ophthalmic Epidemiol. 1996;3:135-41.

19. Nguyen RL, Raja SC, Traboulsi EI. Screening relatives of patients with familial chronic open angle glaucoma. Ophthalmology. 2000;107:1294-7. 